\title{
Conhecimento acerca do Câncer Bucal e Atitudes frente à sua Etiologia e Prevenção em um Grupo de Horticultores de Teresina (PI)
}

Knowledge about Oral Cancer and Attitudes towards its Etiology and Prevention in a Group of Gardeners from Teresina (PI)

El Conocimiento acerca del Cáncer Oral y las Actitudes en cuanto a su Etiología y la Prevención en un Grupo de Horticultores de Teresina (PI)

\author{
Lumena Raquel de Brito Souza' , Kayze Duarte Ferraz², Nayla Silva Pereira ${ }^{3}$, Márcia Valéria Martins ${ }^{4}$
}

\section{Resumo}

Introduçáo: O câncer bucal é uma doença crônica de origem multifatorial. Sua prevenção e diagnóstico precoce são as melhores formas de aumento da sobrevida. Objetivo: Avaliar o nível de informação acerca do câncer bucal e as atitudes frente aos fatores etiológicos e medidas preventivas adotadas por um grupo de horticultores, em Teresina-PI. Método: Os sujeitos do estudo foram 111 horticultores voluntários, pertencentes a seis hortas comunitárias da Zona Leste da cidade de Teresina-Pi. Foi aplicado um formulário constituído de 26 perguntas abertas e fechadas referentes ao perfil sociodemográfico dos sujeitos e ao conhecimento e atitudes frente aos fatores de risco e medidas preventivas relacionadas ao câncer, em especial ao câncer de boca. Os dados foram analisados através de análise de frequências, estatísticas descritiva e inferencial (teste qui-quadrado) com nível de confiança de $95 \%$ ( $\mathrm{p}<0,05)$. Resultados: Observouse que, dos 111 entrevistados, $93(83,8 \%)$ já ouviram falar sobre câncer bucal, $80(72,1 \%)$ acharam que há cura e 72 $(64,9 \%)$ que existe vacina contra o mesmo; dentre os fatores de risco, $103(92,8 \%)$ relataram que se expóem aos raios solares, sendo a maioria (86,5\%) exposta diariamente; e, entre as medidas preventivas mais utilizadas, está o uso de chapéu/boné (46,8\%). Conclusão: A maior parte dos entrevistados já ouviu falar sobre o câncer bucal, mas não o conhece detalhadamente, muitos já haviam entrado em contato com algum fator de risco durante sua vida, sendo o mais frequente a exposição solar; e a maioria utiliza pelo menos uma medida preventiva.

Palavras-chave: Neoplasias Bucais/prevenção \& controle; Fatores de Risco; Conhecimentos, Atitudes e Prática em Saúde

\footnotetext{
${ }^{1}$ Acadêmica do Curso de Odontologia da Faculdade Integral Diferencial (FACID).

${ }^{2}$ Acadêmica do Curso de Odontologia da FACID.

${ }^{3}$ Acadêmica do Curso de Odontologia da FACID.

${ }^{4}$ Professora de Odontologia da FACID. Doutora em Biopatologia Bucal. Área de concentração em Radiologia Odontológica.

Endereço para correspondência: Rua Juiz Joâo Almeida, 1.774 - Bairro Ininga. Teresina (PI), Brasil. CEP: 64049-650. E-mail: lumenaraquel@hotmail
} 


\section{INTRODUÇÃO}

A etiologia do câncer oral é uma somatória de fatores carcinógenos que podem levar ao aparecimento da doença, entre eles, os principais são de origem extrínseca, tais como o fumo e o álcool, além da exposição crônica à radiação solar, nos casos situados em lábio ${ }^{1}$. A exposição aos raios solares ultravioleta (UV) causa sérios danos celulares tanto no epitélio quanto no tecido conjuntivo subjacente, e, excessivamente em contato direto com o lábio inferior, aumenta o risco de desenvolvimento do carcinoma ${ }^{2}$.

O conhecimento dos fatores carcinogênicos permite atuar sobre a relaçáo causa-efeito de agentes causais, assim como selecionar quais pacientes têm maiores probabilidades de desenvolver um tumor específico e atuar em uma etapa precoce ${ }^{1}$. As informaçóes sobre esses fatores constituem a base para uma prevenção efetiva da doença, que precisa ser direcionada para jovens, idosos e os menos instruídos ${ }^{3}$.

Apesar da alta prevalência do câncer oral, poucas campanhas de prevenção foram desenvolvidas no Brasil como uma ação nacional, visando à educação populacional, abordando de forma ampla e irrestrita suas causas, formas de prevenção e de diagnóstico precoce ${ }^{1}$. Por isso, a maioria dos casos da doença é detectada em fase avançada, na qual o tratamento revela-se de alto custo econômico e social, com prognóstico desfavorável, uma vez que essa doença pode levar à morte, invalidez e deformidades ${ }^{4}$.

A literatura da área nos mostra a existência de uma grande lacuna de informaçóes específicas para as diferentes camadas da populaçáo sobre o conhecimento acerca do câncer bucal, principalmente naquelas expostas a fatores de risco, assim como a respeito de seus fatores etiológicos e medidas preventivas adotadas em relação a essa doença. Em decorrência da escassez de estudos no Brasil que se referem ao assunto em questão e da relevância do tema, surgiu o interesse de realizar o presente trabalho, com objetivo de avaliar o nível de informação acerca do câncer bucal e as atitudes frente aos fatores etiológicos e medidas preventivas adotadas por um grupo de horticultores, em Teresina-PI.

\section{MÉTODO}

Previamente à realização do presente trabalho, o respectivo projeto foi submetido ao Comitê de Ética em Pesquisa da Faculdade Integral Diferencial (FACID) e aprovado sob protocolo de número 198/10. Em paralelo, foi obtida a autorização da Prefeitura Municipal de Teresina-PI, através da Superintendência de Desenvolvimento Rural, para realização da pesquisa em algumas hortas comunitárias.
A pesquisa, de caráter transversal e descritivo, foi realizada em seis hortas comunitárias situadas na Zona Leste de Teresina-PI, devido ao maior número de hortas comunitárias nessa região. A população em análise era composta de 265 famílias, sendo entrevistado um membro de cada família, perfazendo um total de 265 indivíduos. No entanto, o estudo foi realizado com uma amostra probabilística de 111 horticultores, definida utilizando-se a fórmula de erro amostral da população finita, tendo como erro amostral aceitável $1 \%$.

Os sujeitos da pesquisa foram os horticultores registrados na Prefeitura Municipal de Teresina-PI, maiores de 18 anos, e assinaram o Termo de Consentimento Livre e Esclarecido. Entre os que atendiam a esses critérios, os participantes foram escolhidos de maneira casual simples e voluntariamente.

Esse grupo populacional foi escolhido para o referente trabalho devido à sua ocupação profissional, estando em contato direto com, pelo menos, um fator de risco, por ocuparem uma baixa posiçáo social, por não terem acesso a uma boa escolaridade e por estarem disponíveis em diferentes áreas da cidade.

A coleta de dados foi realizada mediante a aplicação de um formulário (Apêndice A), sob forma de entrevista, realizado por uma única pesquisadora, sendo essa aluna regularmente matriculada no $\mathrm{X}$ bloco do curso de Odontologia da FACID e autora do presente trabalho.

O formulário aplicado foi constituído de 26 perguntas abertas e fechadas referentes ao perfil sociodemográfico dos sujeitos e ao conhecimento e atitudes frente aos fatores de risco e medidas preventivas relacionados ao câncer e, em especial, ao câncer de boca.

Após a coleta dos dados, esses foram tabulados no software Excel $^{\oplus}$ for Windows ${ }^{\ominus}$ e submetidos à análise estatística no programa SPSS ${ }^{\circledR} 17.0$ para Windows ${ }^{\ominus}$. A seguir, foram analisadas, através de estatística descritiva (média e desvio-padrão), frequências (absoluta e relativa) e estatística inferencial (teste qui-quadrado). O intervalo de confiança admitido foi de $95 \%(\mathrm{p}<0,05)$ e os resultados obtidos foram apresentados através de tabelas e gráficos no Microsoft Word ${ }^{\oplus}$.

\section{RESULTADOS E DISCUSSÃO}

Em relação ao perfil sociodemográfico, expresso na Tabela 1, a maioria dos horticultores era de cor parda, característica observada de acordo com as diretrizes do IBGE, possuía o ensino fundamental incompleto e baixa renda (tinha como renda mensal menos de um salário mínimo). Foi constatada a predominância do gênero feminino, contrastando com o resultado de outros autores, os quais relataram as atividades domésticas domiciliares 
prevalentes para esse gênero e as atividades rurais para o gênero masculino ${ }^{5}$. Como essa doença tem predomínio marcante no gênero masculino e em pessoas brancas ${ }^{6-7}$, a maioria pesquisada no presente trabalho encontra-se fora desses parâmetros.

Assim como os diversos outros tipos, o câncer de boca é uma doença que acomete, comumente, pessoas de meia-idade, aquelas acima dos 40 anos. A idade média da populaçáo pesquisada foi de 52,7 anos com desvio padráo de 11,1 anos (idade mínima de 25 anos e máxima de 78 anos), estando mais propícios ao desenvolvimento dessa patologia ${ }^{1,5,8-9}$.

Tabela 1. Número absoluto e percentual de horticultores segundo suas características sociodemográficas

\begin{tabular}{|c|c|c|}
\hline Variável & $\mathbf{N}$ & $\%$ \\
\hline \multicolumn{3}{|l|}{ Gênero } \\
\hline Feminino & 66 & 59,5 \\
\hline Masculino & 45 & 40,5 \\
\hline \multicolumn{3}{|l|}{ Cor } \\
\hline Branca & 11 & 9,9 \\
\hline Preta & 38 & 34,2 \\
\hline Parda & 58 & 52,3 \\
\hline Amarela & 4 & 3,6 \\
\hline \multicolumn{3}{|l|}{ Escolaridade } \\
\hline Analfabeto & 33 & 29,7 \\
\hline Ensino fundamental completo & 19 & 17,1 \\
\hline Ensino fundamental incompleto & 51 & 45,9 \\
\hline Ensino médio completo & 6 & 5,5 \\
\hline Ensino superior incompleto & 1 & 0,9 \\
\hline Ensino superior completo & 1 & 0,9 \\
\hline \multicolumn{3}{|l|}{ Renda familiar } \\
\hline < 1 salário mínimo & 46 & 41,4 \\
\hline 1 salário mínimo & 34 & 30,6 \\
\hline 1>3 salários mínimos & 31 & 28,0 \\
\hline
\end{tabular}

De acordo com a Tabela 2, observou-se que o conhecimento, acerca do câncer bucal, no grupo estudado, foi baixo. No entanto, após aplicação do teste qui-quadrado $(p<0,01)$, o nível de conhecimento foi estatisticamente superior entre os indivíduos que possuíam o ensino fundamental completo, quando comparados com os analfabetos. A análise com os demais níveis de escolaridade (Tabela 1) não pôde ser aprofundada devido ao pequeno número de representantes nessas categorias, mostrando que as diferenças entre os mesmos não são estatisticamente significativas entre si $(p<0,254)$. Quanto ao fato de já ter ouvido falar sobre o câncer de boca, existe uma diferença estatisticamente significativa entre as pessoas que responderam sim e as que responderam não $(\mathrm{p}<0,01)$. Assim como em outra pesquisa, a maioria pesquisada já ouviu falar na doença, por ser uma questão de conhecimento público, difundida pelos meios de comunicaçáo como a televisáo ${ }^{10}$, mas não demonstram conhecimento algum sobre a patologia, também confirmado por outros autores ${ }^{3,11}$.

Quando questionados sobre o risco de contágio do câncer de boca e a probabilidade de sua cura, houve uma diferença estatisticamente significante $(\mathrm{p}<0,01)$ entre aqueles que responderam sim, a maioria, e os que responderam não. Entretanto, não há evidências científicas de que o contato próximo, o ato sexual, o beijo, o ato de compartilhar talheres, entre outras atividades possam transmitir qualquer tipo de câncer ${ }^{12}$.

Em relação à cura, a detecção precoce dos sinais da doença faz com que haja a busca do tratamento na fase inicial, o que proporcionará a reabilitação do paciente na maioria dos casos, reduzindo também as sequelas funcionais e estéticas. Caso não seja detectado ou realizado um tratamento precocemente, há o risco de maiores mutilaçôes ou até mesmo de vir a óbito ${ }^{7}$.

As demais variáveis da Tabela 2 não mostraram significância estatística entre aqueles que responderam sim e os que responderam não, o que demonstrou não haver um nível de conscientização elevado, caracterizando acesso à informaçáo aleatória. O que acontece, por exemplo, sobre a especulação de haver uma vacina contra o câncer, que ainda está em fase experimental ${ }^{7}$.

Tabela 2. Distribuição do número absoluto e percentual das respostas relacionadas ao conhecimento geral sobre o câncer bucal

\begin{tabular}{|c|c|c|c|c|}
\hline \multirow{2}{*}{ Variável } & $\mathbf{N}$ & $\%$ & $\mathbf{N}$ & $\%$ \\
\hline & \multicolumn{2}{|c|}{ SIM } & \multicolumn{2}{|c|}{ NÃO } \\
\hline Já ouviu falar sobre o câncer de boca? & 93 & 83,8 & 18 & 16,2 \\
\hline Há risco de contágio da doença? & 72 & 64,9 & 39 & 35,1 \\
\hline Há cura? & 80 & 72,1 & 31 & 27,9 \\
\hline Há vacina? & 56 & 50,5 & 55 & 49,5 \\
\hline Conhece o autoexame da boca? & 47 & 42,3 & 64 & 57,7 \\
\hline
\end{tabular}


O câncer de boca é uma doença de fácil diagnóstico, bastando uma inspeção detalhada da cavidade oral, seja por parte de um profissional ou do próprio indivíduo. O autoexame da boca, ao contrário do autoexame da mama, ainda é pouco difundido, sendo desconhecido pela maior parte da população. Uma hipótese para esse fato é que os cirurgiôes-dentistas não costumam ensinálo e nem o realizam em busca de lesôes cancerizáveis na prática clínica, por não saberem fazer, por não acharem necessário, ou por não receberem honorários para isso ${ }^{13}$. Isso demonstra falta de responsabilidade, ou ainda, de comprometimento do profissional para com o paciente.

É importante a necessidade da consciência de tornarse rotineiro o exame para prevenir ou diagnosticar o câncer de boca, realizando-se esforços para informar à populaçáo sobre os agentes carcinogênicos, como evitá-los e preveni-los. Todavia, o comportamento preventivo está ligado a fatores sociais, psicológicos, ambientais e, ainda, culturais, como as crendices, podendo influenciar positivo ou negativamente, dependendo do predomínio desses fatores no estilo de vida dos indivíduos ${ }^{14}$.

$\mathrm{O}$ retardo no diagnóstico prende-se a diversos fatores, entre eles, a desinformação da população leiga, que mantém crenças ultrapassadas e negativas sobre essa patologia e seu prognóstico; a falta de alerta dos profissionais da saúde para o diagnóstico precoce dos casos; e a falta de rotinas abrangentes programadas nos serviços de saúde públicos e privados, que favoreçam a detecção do câncer ${ }^{15}$.

Os casos de câncer estão relacionados a fatores ambientais, ou seja, aos hábitos ocupacionais, sociais e culturais, e, além desses, ainda estâo associados aos fatores genéticos. Quanto ao hábito de fumar, ao consumo de bebida alcoólica e à exposição ao sol (Tabela 3), as diferenças observadas nos padróes de respostas foram estatisticamente significativas, entre os horticultores que responderam sim e os que responderam não $(\mathrm{p}<0,01)$.
Atualmente, são muito divulgadas as consequências do hábito de fumar e sua associação com diversos tipos de doenças, inclusive o câncer oral. O tabaco atua como um potente fator de risco ${ }^{1-2,8-11,13,16-19}$. Talvez, por esses motivos, pôde-se observar um reduzido número de fumantes na amostra estudada, conforme observado na Tabela 3.

O consumo de álcool também é um fator predisponente ao desenvolvimento do câncer bucal ${ }^{1,8-9,11,13,16,19-20}$. Apesar de ter tornado-se um hábito frequente nas reuniôes sociais, apenas uma pequena parcela dos pesquisados consumia bebida alcoólica (Tabela 3), o que pode ser explicado pela predominância do gênero feminino, mas que não pôde ser provado devido à pequena diferença entre mulheres $\mathrm{e}$ homens que consumiam esse tipo de bebida.

A bebida mais consumida entre os entrevistados foi a cerveja $(26,1 \%)$, que tem uma forte associação com o câncer de boca, juntamente com o licor ${ }^{16}$. Em seguida, veio a cachaça $(15,3 \%)$, bebida mais consumida em outra pesquisa $^{20}$.

Muito embora sejam conhecidos outros fatores de risco associados ao câncer de boca, como o uso de próteses mal adaptadas e a presença de dentes em mau estado (Tabela 3 ), que podem provocar irritação crônica na mucosa ${ }^{2}$, há uma escassez de trabalhos na literatura que relatem esses fatores ${ }^{19}$.

$O$ fator genético, apesar de exercer um importante papel na oncogênese, ou o fato de já ter desenvolvido um câncer previamente não são fatores exclusivos, ou seja, raramente essa doença desenvolve-se apenas por esses motivos. Somente cerca de $5 \%$ a $10 \%$ dos casos de câncer são herdados .

Há uma forte relação entre a exposição solar UVB e a incidência (maior no sexo feminino - 83\%) e a mortalidade (maior no sexo masculino - 79\%) do câncer da cavidade oral ${ }^{21}$, fator este a que os membros desse grupo estão mais expostos, conforme observado na Tabela 3.

O desenvolvimento do câncer oral náo depende apenas do contato com os fatores de risco, pois a doença pode

Tabela 3. Distribuição do número absoluto e percentual das respostas relacionadas a comportamentos de risco para o desenvolvimento do câncer bucal

\begin{tabular}{|c|c|c|c|c|}
\hline \multirow{2}{*}{ Variável } & $\mathbf{N}$ & $\%$ & $\mathbf{N}$ & $\%$ \\
\hline & \multicolumn{2}{|c|}{ SIM } & \multicolumn{2}{|c|}{ NÃO } \\
\hline Tem o hábito de fumar? & 27 & 24,3 & 84 & 75,7 \\
\hline Já teve o hábito de fumar? & 29 & 26,1 & 55 & 49,5 \\
\hline Consome bebida alcoólica? & 35 & 31,5 & 76 & 68,5 \\
\hline Já consumiu bebida alcoólica? & 23 & 19,9 & 53 & 47,6 \\
\hline Faz uso de prótese? & 75 & 67,6 & 36 & 32,4 \\
\hline Possui dentes em mau estado? & 50 & 45 & 61 & 55 \\
\hline Já foi acometido pelo câncer? & 3 & 2,7 & 108 & 97,3 \\
\hline Expõe-se muito ao sol? & 103 & 92,8 & 8 & 7,2 \\
\hline
\end{tabular}


apresentar-se após vários anos de exposição contínua a esses fatores. Entretanto, depende do tempo e frequência de contato com o fator etiológico, tendo um efeito dosedependente ${ }^{3,16,20}$. Aqueles que estão em contato há mais tempo são mais propensos a desenvolver a doença.

A prevenção é o melhor meio para se proteger dos desastres causados pelos diversos tipos de câncer. Dos 93 entrevistados que afirmaram já ter ouvido falar sobre o câncer bucal (Tabela 2), 78 utilizavam algum método preventivo, fato confirmado após aplicação do teste quiquadrado $(\mathrm{p}<0,01)$. De acordo com a Tabela 4 , houve uma preferência dos indivíduos pela proteção conferida por chapéu/boné em detrimento das outras formas de proteção específicas para radiação solar $(\mathrm{p}<0,01)$.

O autoexame da boca, em especial, é de fácil realização e de baixo custo, podendo ser realizado em frente ao espelho de casa. Entretanto, não é uma prática aplicada. Mesmo os sujeitos que já ouviram falar sobre essa medida preventiva (Tabela 2 ), o que equivale a uma pequena parcela da população, não a realiza, conforme observado na Tabela 4, resultado este também encontrado por outros pesquisadores ${ }^{3}$.

O uso de batom é uma forma de proteção utilizada pelas mulheres. Entretanto, entre as 66 pesquisadas, apenas 29 faziam uso; e, dessas, 27, uma quantidade significativa $(\mathrm{p}<0,01)$, afirmaram conhecer o câncer de boca.

O consumo de frutas e verduras é outra forma de prevenção que pode ser adquirida por todos, sendo esse inversamente proporcional ao desenvolvimento do câncer de boca ${ }^{22}$. Entretanto, apesar de trabalharem com horticultura, nem todos os pesquisados consumiam frutas e verduras. Essa população em específico deve utilizar métodos preventivos adicionais, como o uso de camisas de mangas compridas, chapéus ou bonés, além de não se expor ao sol das 10 horas às 16 horas. $\mathrm{O}$ uso do protetor solar corporal e labial seria de suma importância, mas não é economicamente viável aos horticultores.

A visita ao dentista (Tabela 4) deve ser realizada de seis em seis meses, o que não foi demonstrado na pesquisa, em que $34,2 \%$ da população relataram ter procurado esse profissional há mais de cinco anos, e por motivo de dor $(44,1 \%)$. Esses dados confirmam o pensamento de que ainda vivemos na era da assistência curativa. $\mathrm{O}$ modelo preventivo não atingiu a grande maioria, em especial aqueles com condiçôes socioeconômicas mais baixas ${ }^{14}$.

\section{CONCLUSÃO}

Após análise dos resultados obtidos, foi possível concluir que, na amostra estudada, apesar de a maioria já ter ouvido falar do câncer de boca, quando questionados de forma mais detalhada, apresentavam um conhecimento superficial e fragmentado. Observou-se que apenas uma minoria possuía conhecimento aprofundado acerca da referida patologia. Todos os pesquisados já haviam entrado em contato com algum fator de risco durante sua vida, no qual o mais citado foi a exposição solar, devido à ocupação profissional da população pesquisada. Em relação à adoção de medidas preventivas, a maioria utiliza pelo menos uma, mesmo que empiricamente.

Tabela 4. Distribuição do número absoluto e percentual das respostas relacionadas às medidas preventivas para o desenvolvimento do câncer bucal, utilizadas pela população pesquisada

\begin{tabular}{|c|c|c|c|c|}
\hline \multirow{2}{*}{ Variável } & $\mathbf{N}$ & $\%$ & $\mathbf{N}$ & $\%$ \\
\hline & \multicolumn{2}{|c|}{ SIM } & \multicolumn{2}{|c|}{ NÃO } \\
\hline Realiza o autoexame da boca? & 8 & 7,2 & 103 & 92,8 \\
\hline Faz uso de batom? & 29 & 26,1 & 82 & 73,9 \\
\hline Consome frutas e verduras & 104 & 93,7 & 7 & 6,3 \\
\hline \multicolumn{5}{|l|}{ Tempo da última visita ao dentista } \\
\hline Menos de 1 ano & 29 & 26,1 & 82 & 73,9 \\
\hline 1-2 anos & 26 & 23,4 & 85 & 76,6 \\
\hline $3-5$ anos & 17 & 15,3 & 94 & 84,7 \\
\hline Mais de 5 anos & 38 & 34,2 & 73 & 65,8 \\
\hline \multicolumn{5}{|l|}{ Proteção específica } \\
\hline Não se protege & 18 & 16,2 & 93 & 83,8 \\
\hline Filtro solar & 23 & 20,7 & 88 & 79,3 \\
\hline Chapéu/boné & 52 & 46,8 & 59 & 53,2 \\
\hline Camisa com mangas compridas & 16 & 14,4 & 95 & 85,6 \\
\hline
\end{tabular}


A análise realizada nos remete a pensar acerca da necessidade da populaçáo ter acesso a informaçóes mais consistentes sobre a referida doença, englobando os fatores etiológicos e medidas preventivas, o que pode ser realizado por meio de campanhas educacionais e de atuaçóes dos profissionais da saúde, principalmente os cirurgiōesdentistas e, preferencialmente, nos grupos populacionais considerados de risco.

\section{CONTRIBUIÇÕES}

Lumena Raquel de Brito Souza contribuiu na concepçáo e planejamento do projeto de pesquisa, obtenção, análise e interpretação dos dados e redação do texto; Kayze Duarte Ferraz contribuiu na obtenção de dados; Nayla Silva Pereira contribuiu na obtençáo de dados; Valéria Martins contribuiu no planejamento do projeto de pesquisa, análise e interpretação dos dados e revisão crítica.

\section{Declaraçáo de Conflito de Interesses: Nada a Declarar.}

\section{REFERÊNCIAS}

1. Martins MAT, Marques FGOA, Pavesi VCS, Romão MMA, Lascala CA, Martins MD. Avaliação do conhecimento sobre o câncer bucal entre universitários. Rev bras cir cabeça pescoço. 2008;37(4):191-7.

2. Prado BN, Passarelli DHC. Uma nova visão sobre prevenção do câncer bucal no consultório odontológico. Rev odontol Univ Cid São Paulo. 2009;21(1):79-85.

3. Mauricio HA, Matos FCM, Guimarães TMR. Conhecimentos, atitudes e práticas sobre câncer de boca da comunidade atendida pelo PSF de São Sebastião do Umbuzeiro/PB. Rev bras cir cabeça pescoço. 2009;38(1):10-4.

4. Falcão MML, Alves TDB, Freitas VS, Coelho TCB. Conhecimento dos cirurgiōes-dentistas em relação ao câncer bucal. RGO. 2010;58(1):27-33.

5. Alvarenga LM, Ruiz MT, Pavarino-Bertelli EC, Ruback MJC, Maniglia JV, Goloni-Bertollo EM. Avaliação epidemiológica de pacientes com câncer de cabeça e pescoço em um hospital universitário do noroeste do estado de São Paulo. Rev Bras Otorrinolaringol. 2008;74(1):68-73.

6. Santos GL, Freitas VS, Andrade MC, Oliveira MC. Fumo e álcool como fatores de risco para o câncer bucal. Odontol clín-cient. 2010;9(2):131-3.

7. Instituto Nacional de Câncer José Alencar Gomes da Silva. O que é o câncer? [Internet]. [citado 2012 jan 13]. Disponível em: http://www.inca.gov.br/conteudo_view. asp?id $=322$

8. Oliveira JC, Moreira MAR, Martins E, Bandeira e Silva CM, Mineiro MS, Souza EC, et al. A exposição ocupacional como fator de risco no câncer de cavidade oral e orofaringe no Estado de Goiás. Rev bras cir cabeça pescoço. 2008;37(2):82-7.

9. Melo LC, Silva MC, Bernardo JMP, Marques EB, Leite ICG. Perfil epidemiológico de casos incidentes de câncer de boca e faringe. RGO. 2010;58(3):351-5.

10. Lima AAS, França BHS, Ignácio SA, Baioni CS. Conhecimento de alunos universitários sobre câncer bucal. Rev bras cancerol. 2005;51(4):283-8.

11. Vidal AKL, Tenório APS, Brito BHG, Oliveira TBT, Pessoa ID. Conhecimento de escolares do sertão Pernambucano sobre o câncer de boca. Pesqui bras odontopediatria clín integr. 2009;9(3):283-8.

12. American Cancer Society. Is cancer contagious? [Internet]. [Atlanta: American Cancer Society]; 2010 [cited 2012 Jan 13]. Available from: http://www.cancer. org/Cancer/CancerBasics/is-cancer-contagious

13. Gajendra S, Cruz GD, Kumar JV. Oral cancer prevention and early detection: knowledge, practices, and opinions of oral health care providers in New York State. J Cancer Educ. 2006;21(3):157-62.

14. Cestari MEW, Zago MMF. A prevenção do câncer e a promoção da saúde: um desafio para o Século XXI. Rev Bras Enferm. 2005;58(2):218-21.

15. Cimardi ACBS, Fernandes APS. Câncer bucal: a prática e a realidade clínica dos cirurgiōes-dentistas de Santa Catarina. RFO UPF. 2009;14(2):99-104.

16. Morse DE, Psoter WJ, Cleveland D, Cohen D, MohitTabatabai M, Kosis DL, et al. Smoking and drinking in relation to oral cancer and oral epithelial dysplasia. Cancer Causes Control. 2007;18(9):919-29.

17. Bunnell A, Pettit N, Reddout N, Sharma K, O'Malley S, Chino M, et al. Analysis of primary risk factors for oral cancer from select US states with increasing rates. Tob Induc Dis. 2010;8:5.

18. Devadiga A, Prasad KV. Knowledge about oral cancer in adults attending a Dental Hospital in India. Asian Pac J Cancer Prev. 2010;11(6):1609-13.

19. Zygogianni AG, Kyrgias G, Karakitsos P, Psyrri A, Kouvaris J, Kelekis N, et al. Oral squamous cell cancer: early detection and the role of alcohol and smoking. Head Neck Oncol. 2011;3:2.

20. Abdo EN, Santos PJB, Mendonça LL, Garrocho AA, Aguiar MCF. Álcool e fumo como determinantes da localização do carcinoma epidermóide de boca. Arq odontol. 2005;41(3):226-34.

21. Boscoe FP, Schymura MJ. Solar ultraviolet-B exposure and cancer incidence and mortality in the United States, 1993-2002. BMC Cancer. 2006;6:264.

22. Toledo AL, Koifman RJ, Koifman S, Marchioni DM. Dietary patterns and risk of oral and pharyngeal cancer: a case-control study in Rio de Janeiro, Brazil. Cad Saúde Pública. 2010;26(1):135-42. 


\section{APÊNDICE A - Formulário de Coleta de Dados}

Local da Pesquisa:

Data:

Nome:

1 - Idade:

2 - Gênero:

(0) Feminino (1) Masculino

3-Cor:
(0) Branca
(1) Preta
(2) Parda
(3) Amarela ( ) Outra

4 - Grau de instruçáo:

(0) Ensino Fundamental Completo (1) Ensino Fundamental Incompleto (2) Ensino Médio Completo

(3) Ensino Médio Incompleto (4) Ensino Superior Incompleto (5) Ensino Superior Completo (6) Analfabeto

5 - Renda familiar:

(0) Menos de 1 salário (1) 1 salário (2) de 1 a 3 salários (3) mais de 3 salários

6 - Conhece ou já ouviu falar sobre o câncer bucal?

(0) Sim (1) Não

7 - Há risco de contágio para as pessoas que convivem com o portador?

(0) Sim (1) Não

8 - Esta doença tem cura?

(0) Sim (1) Não

9 - Existe vacina?

(0) Sim (1) Não

10 - Conhece o autoexame da boca?

(0) Sim (1) Não

11 - Realiza esse autoexame?

(0) Sim (1) Não

12 - Você fuma?

(0) Sim (1) Não Há quanto tempo?

13 - Já fumou?

(0) Sim (1) Náo Por quanto tempo?

14 - Consome bebida alcoólica?

(0) Sim (1) Não Há quanto tempo?

15 - Já consumiu bebida alcoólica?

(0) Sim (1) Nãoo Por quanto tempo?

16 - Com que frequência você consome bebida alcoólica?

(0) Esporadicamente (1) Diariamente (2) Frequentemente 
17 - Que tipo de bebida você consome?

18 - Usa prótese?

(0) Sim (1) Não

19 - Possui dentes em mau estado?

(0) Sim (1) Não

20 - Já teve câncer?

(0) Sim (1) Não

21 - Se expóe muito ao sol?

(0) Sim (1) Não Frequência?

22 - Qual proteção você usa?

(0) Não se protege (1) Filtro solar (2) Chapéu/boné (3) Camisa com mangas compridas (4) Outras

23 - Usa batom?

(0) Sim (1) Não

24 - Consome frutas e verduras?

(0) Sim (1) Não Frequência?

25 - Quando foi sua última visita ao dentista?

(0) Menos de 1 ano (1) 1-2 anos (2) 3-5 anos (3) Mais de 5 anos

26 - Qual foi o motivo?

(0) Prevenção (1) Dor (2) Cárie (3) Problemas gengivais (4) Prótese (5) Outros 


\section{Abstract}

Introduction: Oral cancer is a chronic disease of multi-factorial origin. Its prevention and early diagnosis are the best ways to increase survival rates. Objective: To assess the level of information about oral cancer and attitudes towards etiological factors and preventive measures adopted by a group of gardeners, in Teresina-Pi. Methods: 111 volunteer gardeners were the subjects of this study, from six community gardens of the Eastern Zone of the city of Teresina-Pi. A form consisting of 26 open and closed questions regarding their demographic profile and knowledge and attitudes towards risk factors and preventive measures related to cancer, in particular oral cancer, was applied. Data were analyzed by frequency analysis, descriptive and inferential statistics (chi-square test) with a confidence level of 95\% ( $\mathrm{p}<0.05)$. Results: It was observed that of the 111 respondents, 93 (83.8\%) had heard of oral cancer, $80(72.1 \%)$ believed that there is cure and $72(64.9 \%)$ reported that there is vaccine against it; among the risk factors, $103(92.8 \%)$ reported that expose themselves to sunlight, the majority $(86.5 \%)$ is daily exposed to it, and among the most preventive measures used is the use of hat/cap (46.8\%). Conclusion: Most respondents have heard about oral cancer, but do not know it in detail, many had already been in contact with some risk factor during their lifetime, of which the most frequent is sun exposure, and most of them have used at least one of the preventive measures.

Key words: Mouth Neoplasms/prevention \& control; Risk Factors; Health Knowledge, Attitudes, Practice

\section{Resumen}

Introducción: El cáncer oral es una enfermedad crónica de origen multifactorial. Su prevención y el diagnóstico precoz son las mejores formas de aumentar la supervivencia. Objetivo: evaluar el nivel de información sobre el cáncer oral y las actitudes hacia los factores etiológicos y medidas preventivas adoptadas por un grupo de horticultores en TeresinaPI. Método: los sujetos del estudio eran 111 horticultores voluntarios, pertenecientes a seis huertas comunitarias en la región este de la ciudad de Teresina-PI. Fue distribuido un formulario de 26 preguntas abiertas y cerradas sobre el perfil sociodemográfico de los sujetos, su conocimiento y las actitudes hacia los factores de riesgo y medidas preventivas relacionadas al cáncer, especialmente a los cánceres de boca. El análisis de los dados fue llevado a cabo a través de análisis de frecuencias, estadísticas descriptivas y por deducción (test chi-cuadrado) con un nivel de confianza del 95\% ( $\mathrm{p}<0,05)$. Resultados: Se observó que de los 111 entrevistados, 93 (83,8\%) estaban enterados del cáncer oral, $80(72,1 \%)$ creen que hay cura y $72(64,9 \%)$ que existe una vacuna contra ese cáncer; entre los factores de riesgo, 103 $(92,8 \%)$ informaron que se exponen a la luz del sol, y la mayoría (86,5\%) a diario; y entre las medidas preventivas más utilizadas está el uso de sombrero/gorra (46,8\%). Conclusión: La mayoría de los encuestados han oído hablar sobre el cáncer oral, pero no lo conocen en detalle, muchos han estado en contacto con algún factor de riesgo durante su vida, y la exposición al sol el factor más frecuente, y la mayoría utiliza por lo menos una medida preventiva. Palabras clave: Neoplasias de la Boca/prevención \& control; Factores de Riesgo; Conocimientos, Actitudes y Práctica en Salud 